\title{
Comparison of different surgical techniques of sacrococcygeal pilonidal sinus disease
}

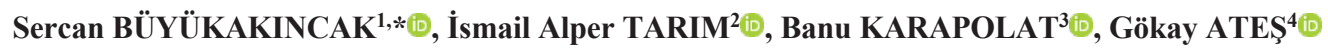 \\ ${ }^{1}$ Department of General Surgery, Akçaabat Haçkalıbaba State Hospital, Trabzon, Turkey \\ ${ }^{2}$ Department of General Surgery, Faculty of Medicine, Ondokuz Mayıs University, Samsun, Turkey \\ ${ }^{3}$ Department of General Surgery, Kanuni Training Hospital, University of Health Sciences, Trabzon, Turkey \\ ${ }^{4}$ Department of Anesthesiology and Reanimation, Akçaabat Haçkalıbaba State Hospital, Trabzon, Turkey
}

\begin{abstract}
\begin{tabular}{ccccc}
\hline Received: 16.12 .2020 & $\bullet$ & Accepted/Published Online: 06.02.2021 & Final Version: 23.04 .2021 \\
\hline
\end{tabular}
\section{Abstract}

Pilonidal sinus, especially in young males, is one of the most common diseases of the sacrococcygeal region. Although many surgical techniques have been described for pilonidal sinus disease (PSD), high recurrence rates and patient satisfaction are still controversial with regard to the ideal treatment modality. In this study, we aimed to compare the complications in Primary excision, Limberg flap, elliptical rotation flap and karydakis techniques used in patients with pilonidal sinus disease. The patients who were diagnosed with Pilonidal sinus and underwent surgery between January 2012 and January 2017 were retrospectively analysed from our hospital database. Patients in whom the Primary repair, Limberg flap, elliptical rotation flap and karydakis flap repair performed were divided into four groups. The groups were compared in terms of their demographic characteristics, length of hospitalization, seroma formation, surgical site infections, wound dehiscence, flap necrosis, sensory loss and recurrence. There were 107 patients in the primary group, 70 in the Limberg flap group, 72 in the elliptical rotation flap group and 45 in the karydakis flap group. There was a statistically significant difference between the groups in terms of the length of hospitalization $(\mathrm{p}<0.001)$. The maximum length of hospitalization period was observed in the Limberg group and was $2.06 \pm 0.95$ days. The minimum duration of hospitalization was 1.00 \pm 0.00 days in the karydakis group. Wound site infection was observed mostly in the primary group with $15 \%$ frequency, whereas this higher ratio was significant when compared with Elliptical Rotation flap and Karydakis flap groups ( $p=0.010,0.024$ respectively). There was no statistically significant difference between the groups in terms of wound dehiscence, flap necrosis, sensory loss and recurrence. There is no consensus on the ideal surgical technique selection for PSD. Our study revealed that wound infection is common in patients undergoing Primary repair and that there is a difference in terms of length of hospitalization for different techniques. We expect the factors we investigate to be a guide for the surgeons.
\end{abstract}

Keywords: pilonidal sinus, postoperative complications, recurrence, surgical treatment

\section{Introduction}

Sacrococcygeal Pilonidal Sinus Disease (PSD), especially in young males, is an inflammatory disease that occurs more commonly in the intergluteal region with acute painful episodes or chronically (Okuş et al., 2013; Sondenaa et al., 1995). The incidence is $0.7 \%$ and is usually in the $15-25$ age group, but can be observed at any age. It is most frequently seen in the period that sex hormones increase after puberty when they affect the pilosebaceous glands and healthy hairs in this region (Sondenaa et al., 1995; Humphries et al., 2010; Duman et al., 2016). It is observed in women at an earlier age, and the possible cause is pubertas praecox. Although the etiology of the disease cannot be determined precisely, it is the most commonly accepted theory that the hair loss from the skin can cause foreign body reaction, chronic irritation and inflammation in the intergluteal region. Risk factors for PSD include obesity, smoking, poor personal hygiene, sedentary lifestyle, family history, deep intergluteal cleft, and excessive body hair in the body (Okuş et al., 2013; Sondenaa et al., 1995; Menteş et al., 2008; Uçar et al., 2015; Vedder, 2006). PSD is often seen as a disease that affects the quality of life adversly in the population of the productive age group, and this leads to labour and material losses. Many conservative and surgical methods have been described in the treatment of PSD, but the standard and optimal surgical method remains controversial (Humphries et al., 2010). The advantages and disadvantages of the treatment modalities, the lack of superiority to each other, led the surgeons to try new methods. Medical treatments include local curettage, phenol injection, silver nitrate applications and electrocauterization of the cavity. Excisionprimary repair, excision-marsupialization, de-epithelialization, excision-open left, Bascom operation, Karydakis operation, VY-Z plasty, Limberg flap and elliptical rotation flap techniques 
vary depending on clinical experience and are frequently used among the surgical techniques (Sondenaa et al., 1995).

In this study, we aimed to compare the complications and recurrence in patients who underwent primary repair, Limberg flap, elliptical rotation flap, and karydakis due to PSD.

\section{Materials and methods}

We retrospectively analysed the patients from our hospital database who were admitted to General Surgery outpatient clinic between January 2012 - January 2017, diagnosed with PSD and underwent 4 different surgical techniques, and contacted them for examination. We divided the cases into four groups according to the type of surgery performed: Primary repair, Limberg flap, elliptical rotation flap and karydakis. Main demographic characteristics such as age and gender, hospitalization, seroma formation, surgical site infections, wound healing, flap necrosis, loss of sensation and relapse database were evaluated with physical examination findings and questioning of cases.

\subsection{Surgical technique}

The patients are hospitalized on the day of surgery and the operative field is shaved. First generation cephalosporin antibiotic prophylaxis is performed 30 minutes before surgery. After spinal anesthesia, prone Jack-knife position is maintained. The plasters from the gluteal region of the cases are adhered to the edges of the operating table, and the intergluteal cleft is exposed and a better view and manipulation environment is created.

\subsection{Primary repair}

The skin was excised by subcutaneous tissues and total excision to the presacral fascia including all sinus tracts up to the sacral fascia was performed. The skin was primarily closed with $2 / 0$ vicryl suture and was passed through the sacral fascia with $2 / 0$ prolene.

\subsection{Limberg and elliptical rotation flap}

In order to reveal the pilonidal sinus cavity, each patient was injected into the methylene blue sinus with the help of an injector. First, the pilonidal cyst was excised totally from the 2 $\mathrm{cm}$ lateral midline by an elliptical or rhombic incision without injuring the pilonidal sinus and without leaving the sinus appendages until the presacral fascia. In the patients who underwent Limberg flap procedure, the skin was deepened to include the skin, subcutaneous tissue and the gluteal muscle fascia where flap will be performed, and to be the continuation of the rhombus and in equal length to the edges of the rhombus and a Limberg flap was prepared. In cases with elliptical rotation flap, the elliptical rotation flap from the right or left gluteal region was prepared to contain the same tissue layers. After bleeding control, the patches which kept the gluteus in traction were opened for ease of closure, the tension in the flaps was removed and following the insertion of the absorbent drain in the defect, the skin was individually sutured with $2 / 0$ vicryl to include the fascia-subcutaneous tissues together. The skin was approximated using 2/0 polypropylene sutures.

\subsection{Karydakis flap technique}

The procedure was initiated with an asymmetric ellipsoid incision. If there was a secondary opening and/or a palpable cyst on the lateral line of the midline, the incision edge was shifted to the lesion. If there was no lesion observed, the incision side was randomly selected. The tissue was removed until the presacral fascia after the incision. Then, a flap extending through the entire incision, $1 \mathrm{~cm}$ below the edge of the midline, $2 \mathrm{~cm}$ inward, was prepared using cautery. The prepared flap was fixed to the other wound edge by the skin and subcutaneous sutures so that the midline was shifted.

\subsection{Statistical analysis}

All statistical data analyses were performed using SPSS 18.0 version package program for Windows. One way ANOVA and chi-square test were used for descriptive statistics and significance of differences. The level of statistical significance was accepted as $\mathrm{p}<0.05$.

\section{Results}

Of the 294 cases, $262(89.12 \%)$ were male and $32(10.88 \%)$ were female. The male/female ratio was 8.19 . The age distribution of the cases is shown in Table 1. The age difference between the groups was between Limberg and karydakis groups (Table 1).

Table 1. The average age of the groups

\begin{tabular}{|l|l|l|l|}
\hline & $\mathrm{N}$ & Mean $\pm \mathrm{SD}$ & $\mathrm{p}$ \\
\hline Primary (Group 1) & 107 & $26.65 \pm 7.37$ & \\
\hline Limberg flap (Group 2) & 70 & $27.50 \pm 7.83$ & \\
\hline $\begin{array}{l}\text { Elliptical rotation flap } \\
\text { (Group 3) }\end{array}$ & 72 & $26.51 \pm 7.28$ & $0,027^{*}$ \\
\hline $\begin{array}{l}\text { Karydakis (Group 4) } \\
\text { Total }\end{array}$ & 45 & $23.56 \pm 3.19$ & \\
\hline
\end{tabular}

The median duration of re-examination of the cases is ( $\min 1.5$ - max 5 ) years. The follow-up of the patient's postoperative outpatient clinic control was $2.17 \pm 0.83$ years.

In terms of gender distribution, there were 94 (87.85\%) males and $13(12.15 \%)$ females in the primary repair group, 60 (85.71\%) males and 10 (14.29\%) females in the Limberg flap group, $64(88.89 \%)$ males and $8(11.11 \%)$ females in the elliptical rotation flap group, $44(97.78 \%)$ males and $1(2.22 \%)$ female in karydakis group. No statistically significant difference was observed between the groups in terms of gender distribution $(\mathrm{p}=0.213)$.

The durations of hospitalization after surgery are indicated in Table 2. There was a statistically significant difference between the groups in terms of the length of hospitalization ( $p$ $<0.001$ ). This difference was between limberg and karydakis group, between limberg and primary group, between karydakis and elliptical rotation group, between karydakis and primary, between elliptical rotation and primary $(p<0.001)$. The length of hospitalization after surgery and their order are shown in Table 2. The maximum length of hospitalization was in Limberg group and the minimum length of hospitalization was in karydakis group (Table 2). 
Table 2. Hospitalization periods

\begin{tabular}{l|c|}
\hline Operation type & Number of days (Mean \pm SD) \\
\hline Primary (Group 1) & $1.41 \pm 0.69$ \\
\hline Limberg flap (Group 2) & $2.06 \pm 0.95$ \\
Elliptical rotation flap (Group 3) & $1.99 \pm 0.91$ \\
Karydakis (Group 4) & $1.00 \pm 0.00$ \\
p value & $p<0.001$
\end{tabular}

When evaluated in terms of complications, the number of seroma cases was $10(9.30 \%)$ in the primary repair group, 5 $(7.10 \%)$ in the Limberg flap group, $2(2.80 \%)$ in the elliptical rotation flap group and $1(2.20 \%)$ in the Karydakis group $(\mathrm{p}=$ $0.199)$. There was no statistically significant difference between the groups in terms of seroma accumulation ( $p=$ 0.199).

The number of infected cases was $16(15.00 \%)$ in the primary group, $5(7.10 \%)$ in the Limberg flap group, $2(2.80 \%)$ in the elliptical rotation flap group and $1(2.20 \%)$ in the karydakis group $(p=0.009)$. There was a difference between group 1 and 4 and between groups 1 and 3 in terms of infection (Table 3). Infection was most common in the primary group and the difference was statistically significant compared to karydakis and elliptical rotation groups, but it was not significant compared to limberg (Table 3 ).

Table 3. The comparisons of $p$ values for the incidence of infection according to the type of operation

\begin{tabular}{l|l|l|l}
\hline & Group 1 & Group 2 & Group 3 \\
\hline Group 2 & 0.155 & & \\
Group 3 & 0.010 & 0.272 & \\
Group 4 & 0.024 & 0.401 & 1.000
\end{tabular}

Wound dehiscence occurred in $5(4.70 \%)$ cases in the primary repair group, $7(10.00 \%)$ in the Limberg flap group, 2 $(2.80 \%)$ in the elliptical rotation flap group and $1(2.20 \%)$ in the Karydakis group. There was no statistically significant difference between the groups $(p=0.166)$. Flap necrosis was not observed in any of the cases.

The sensory loss was $2(1.90 \%)$ in the primary group, 6 $(8.60 \%)$ in the Limberg flap group and $5(6.90 \%)$ in the elliptical rotation flap group; not in the karydakis group. There was no statistically significant difference between the groups $(\mathrm{p}=0.053)$.

Recurrence was seen in primary group $10(9.30 \%)$, in Limberg flap group $3(4.30 \%)$, in elliptical rotation flap group $1(1.40 \%)$ and $1(2.20 \%)$ in karydakis group. There was no statistically significant difference between the groups ( $p=0.075$ ). The median time of recurrences is 8 months.

\section{Discussion}

In our study, pilonidal sinus surgical methods were evaluated in terms of complications; there was no difference between the groups in terms of hospitalization period and wound site infection, while there was no difference between the groups in terms of seroma, wound dehiscence, flap necrosis, sensory loss, and recurrence.

Although PSD has been known for many years and is frequently encountered in clinical settings, there is no consensus in the ideal surgical technique selection to be performed. The frequency of postoperative complications and the variability of recurrence rates have led to many new methods. The long treatment period of the disease causes labour loss and economic losses. The main surgical treatment is excision. Surgeons use different techniques to close the defect after excision. Each method has its own advantages and disadvantages. The common purpose of all methods is to reduce the recurrence of the disease, not to disturb the quality of life, to reduce the length of hospital stay and to return to work early to prevent economic losses.

The incidence of PSD is known as 26/100000 (Okuş et al., 2013; Sondenaa et al., 1995). In this study, the majority of cases operated for PSD consisted of males (262 males $(89.12 \%)$ and 32 females $(10.88 \%) . \mathrm{M} / \mathrm{F}$ ratio was determined as 8,19 . The fact that the average age range is among the $2-3$. Decades coincides with the natural characteristics of PSD (Sondenaa et al., 1995; Humphries et al., 2010; Duman et al., 2016). These findings are consistent with the literature data.

One of the most important details that determines the quality of life in patients operated for PSD is the duration of hospitalization and the other is the duration of work. The duration of hospitalization is shorter in these four methods compared to patients left for secondary recovery. There was a difference between the groups. The maximum hospitalization was in the limberg group. This finding is consistent with literature data (Okuş et al., 2013; Menteş et al., 2008; Uçar et al., 2015).

In our study, there was no statistically significant difference between seroma accumulation in all surgical methods, however, seroma rate was higher in patients who underwent Limberg flap and primary repair.

The feeding of the flaps in PSD surgery is physiologically mediated by macro and microcirculation. Macrocirculation anatomy is used in flap definition and design. Arteriole capillary, venular and arterio-venous anastomoses at the microcirculation level are the location where cellular metabolism occurs and the perfusion is mainly controlled (Vedder, 2006). This circulatory device is partially disrupted by the flap due to Limberg flap with a sharp angle end resulting in a decrease in regional blood circulation. Since the elliptical rotation flap does not have a corner, microcirculation is maintained and a homogeneous feeding is provided throughout the flap. In the light of the fact that seroma accumulation in the flaps, which are generally better fed, will be less, we believe that less seroma was detected in the oval flaps in this study.

Wound site infection is a complication that can be seen postoperatively in PSD cases and has serious consequences. Infection is an important factor in the occurrence of recurrence. Among the factors that cause infection are increased bacterial colonization, the wound area is close to the anal canal, obesity, 
localization difficulty and humidity are considered (Duman et al., 2016). In addition, seroma, hematoma, wound site opening accelerates infection formation (Arslan et al., 2016). The resulting infection may endanger the flap viability, adversely affect the life comfort of the cases, cause reoperations and sometimes lead to painful dressings, which will continue for a long time with the patients being left to secondary healing. The number of infected cases was $5(7.10 \%)$ in Limberg flap group, $1(2.20 \%)$ in karydakis group, $2(2.80 \%)$ in the elliptical rotation flap group and $16(15.00 \%)$ in the primary group $(\mathrm{p}=$ 0.009 ). In our study, the rate of infection was higher in the primary repaired group than in the other groups. Among the flap methods, there was less seroma formation than the elliptical rotation flap limberge and karydakis flap method. It was found statistically significant. Limberg flap method in patients who underwent flap treatment compared to those with elliptical rotation flaps can be explained by above mentioned microcirculation problems related to Limberg flaps and especially by the contamination of their corners due to their closer to the anal canal than the elliptical rotation flaps.

In fact, suturing sharp distal edges in Limberg flaps can lead to ischemia and necrosis in the thin corners due to reduced circulation. In elliptical rotation flap reconstruction, flap viability is better especially in distant corners (Polat et al., 2011).

Postoperative loss of regional sensation was observed in the same number of cases in both flap groups and it was determined that the cases were not large enough to cause any discomfort to the quality of life. The sensory loss was six $(8.60 \%)$ in the Limberg flap group, five $(6.90 \%)$ in the elliptical rotation flap group, and two $(1.90 \%)$ in the primary group, but not in the karydakis group $(\mathrm{p}=0.053)$. We believe that the similarity of pilonidal sinus tissue in both groups is similar in two groups and this results in similar loss rates. Although it is not statistically significant, loss of sensation in elliptical rotation flap is less than limberg method. It is notable that there is no sensory loss in the Karydakis group.

Although it depends on the technique used, the recurrence which is the main problem experienced after PSD surgery is observed $3-46 \%$ in the literature (Sondenaa et al., 1995). In this study, Limberg was seen in three $(4.30 \%)$ cases in Limberg flap group, $1(2.20 \%)$ in karydakis group, one $(1.40 \%)$ in the Elliptical rotation flap group and $10(9.30 \%)$ in the primary group. Recurrences are known to develop especially in the first year. Although it is not statistically significant in relapse rates, we see that the number is higher in the primary group. The excisional surgery with primary closure is one of the common methods used as it is simple and early wound healing is its advantage. However, because the relapse rate is reported to be relatively higher compared to other techniques, the frequency of application seems to be significantly reduced in recent years (Ocakoğlu and Ünal, 2012). Karydakis reported that the method he developed was an easy technique, the suture line remained lateral, had early healing and early return advantages, and the recurrence rate was as low as 1\% (Karydakis, 1973).

The causes of recurrence are generally deep localization and dampness of the region, vacuum effect of hip movements, obesity and bacterial overgrowth due to poor hygiene. Critical points of Limberg flap technique include unwanted cosmetic appearance, necrosis of the flap corner and maceration on the skin in the incision area. We believe that the lower part of the incision in the Limberg flap at the flap and the lower pole of the flap in the intergluteal cleft cause the incision area to remain moist and to create hygiene difficulties due to its closeness to the anal canal, increasing the risk of localized infection and causing wound dehiscence and recurrence. In fact, in many studies carried out in the literature about that, it has been determined that the defect in Limberg flap technique can be closed completely and without tension and therefore the recurrence rates of wound infection in the early period are less and the time to return to daily life is shorter with fast recovery time of the cases (Mentes et al., 2008). We believe that the results obtained here will be more valuable with multicentric, prospective randomized clinical studies with larger populations that will be performed in the future. Although there are many methods in the treatment of PSD, the superiority to each other in terms of the complications and recurrences has not been established clearly.

\section{Conflict of interest}

None to declare.

\section{Acknowledgments}

This research was performed in accordance with, Departments of General Surgery and Anesthesiology and Reanimation, Akçaabat Haçkalıbaba State Hospital, Department of General Surgery, Kanuni Training Hospital, Department of General Surgery.

\section{References}

1. Arslan, S., Karadeniz, E., Ozturk, G., Aydinli, B., Bayraktutan, M.C., Atamanalp, S.S., 2016. Modified Primary Closure Method for the Treatment of Pilonidal Sinus. Eurasian. J. Med. 48, 8489.

2. Duman, K., Gırgın, M., Harlak, A., 2016. Prevalence of sacrococcygeal pilonidal disease in Turkey. Asian J. Surg. 04, 14.

3. Humphries, A.E., Duncan, J.E., 2010. Evaluation and management of pilonidal disease. Surg. Clin. North. Am. 90, $113-24$.

4. Karydakis, G.E., 1973. New approach to the problem of pilonidal sinus. Lancet. 22, 1414-1415.

5. Mentes O, Bagci M, Bilgin T, Ozgul O, Ozdemir M., 2008. Limberg flap procedure for pilonidal sinus disease: results of 353 patients. Langenbecks Arch. Surg. 393,185-189.

6. Ocakoğlu, A., Ünal, E., 2012. The comparison of lay open, karydakis flap and limberg flap techniques in pilonidal sinus surgery. Haydarpaşa Numune Eğitim ve Araştırma Hastanesi Tip Dergisi. 52(2), 74-78.

7. Okuş, A., Karahan, Eryılmaz, M.A., Ay, A.S., 2013. Pilonidal hastalığın toplumda görülme sıklığı, yaşa ve cinsiyete göre 
dağılımı (Erken Sonuçlarımız). Selçuk Tıp Derg. 29 (3), 120122 .

8. Polat, C., Gungor, B., Karagul, S., Buyukakincak, S., Topgul, K., Erzurumlu, K., 2011. Is oval flap reconstruction a good modification for treating pilonidal sinuses? Am. J. Surg. 201, 192.

9. Søndenaa, K., Andersen, E., Nesvik, I., Søreide, J.A., 1995. Patient characteristics and symptoms in chronic pilonidal sinus disease. Int. J. Colorectal Dis. 10(1), 39-42.

10. Uçar, A.D., Cartı, E.B., Oymacı, E., Sarı, E., Yakan, S., Yıldırım, M., Erkan, N., 2015. N.Recurrents pilonidal sinus disease surgery: Is it second primary or reoperative surgery? Ulus. Cerrahi Derg. 32, 162-167.

11. Vedder, N.B., 2006. Flap physiology. Mathes Plastic Surgery, 2nd edition. Philadelphia: Saunders Elsevier Inc, 1; 483-506. 